\title{
Smart Local Backup Protection for Smart Substation
}

\author{
M. N. Dazahra' ${ }^{1}$ F. Elmariami ${ }^{2}$ A. Belfqih ${ }^{3}$, J. Boukherouaa ${ }^{4}$ \\ Department of Electrical Networks and Static Converters, National superior School of Electricity and Mechanics, \\ Morocco
}

\begin{tabular}{|c|c|}
\hline Article Info & ABSTRACT \\
\hline $\begin{array}{l}\text { Article history: } \\
\text { Received Apr 11, } 2017 \\
\text { Revised Apr 28, } 2017 \\
\text { Accepted Jun 17, } 2017\end{array}$ & $\begin{array}{l}\text { This paper presents a novel smart local backup protection SLBP used for the } \\
\text { support and backup of the protective relays in a smart substation. The } \\
\text { proposed SLBP is based on the IEC } 61850 \text { standards and the concept consists } \\
\text { of the acquisition of Generic Object Oriented Substation Event GOOSE used } \\
\text { for tripping and interlocks exchange between Intelligent Equipment Devices } \\
\text { IEDs and the reading of the Sampled Value SV existing in the process bus }\end{array}$ \\
\hline $\begin{array}{l}\text { Keywords: } \\
\text { Backup protection } \\
\text { GOOSE } \\
\text { IEC61850 } \\
\text { Process bus } \\
\text { Smart Substation }\end{array}$ & $\begin{array}{l}\text { coming from Mergin Units MU or Non-Conventional Instrument } \\
\text { Transformers NCIT. Several logical schemes to protect different zones of the } \\
\text { substation are presented and how can be integrated using data in the } \\
\text { substation automation system. The SLBP was developed using an open } \\
\text { source library and free operating system. Moreover, a low cost prototype is } \\
\text { presented in order to evaluate the efficiency and the operation of the SLBP } \\
\text { under diverse scenarios of the proposed logical protective schemes such us } \\
\text { breaker failure and overcurrent protection. }\end{array}$ \\
\hline & $\begin{array}{r}\text { Copyright } \odot 2017 \text { Institute of Advanced Engineering and Science. } \\
\text { All rights reserved. }\end{array}$ \\
\hline \multicolumn{2}{|l|}{ Corresponding Author: } \\
\hline \multicolumn{2}{|c|}{$\begin{array}{l}\text { Dazahra Mohamed Nouh, } \\
\text { Department of Electrical Networks and Static Converters, } \\
\text { National superior School of Electricity and Mechanics, } \\
\text { Road El Jadida, Km 7, BP: } 8118 \text {, Oasis - Casablanca, Morocco. } \\
\text { Email: m.n.dazahra@gmail.com }\end{array}$} \\
\hline
\end{tabular}

\section{INTRODUCTION}

The power systems become more loaded and complex especially with the energy and environmental challenges of the 21th century. The majority of power systems operate within their limits, therefore; a simple fault in the electrical power system which is not isolated at right time or a trip that doesn't respect selectivity can cause a blackout to the entire grid example of the blackout of Northeast United States and Canada in 14 August 2003 and the blackout of Italy in 28 September 200 [1].

Nowadays, substations use numerical protective relays for the protection of the primary equipment of the substation, each relay contains several protection functions such as overcurrent, overvoltage and distance protection. In the most cases the relay works independently from other relays existing in the substation, the relays trip after detecting a fault by respecting setting of thre shold and time. This traditional concept suffers from several weakness of redundancy and reliability, because a mal operation of a relay such as a no tripping after a fault, a no respect of setting or a problem in the relay itself can cause severe damages to the substations. Thus, a necessity of backup and hierarchical structure of the protections [2].

With the integration of smart substations many concept of protection has been presented in literature such as wide area protection [3],[4] which protects a certain area of the grid nevertheless these concepts need a robust communication infrastructure which is not available in most cases. Furthermore, some backup Protections used to support the protection of substation based on direction comparison principle and current differential protection principle were presented [5-8] however, those algorithms are complexes to be implemented in SAS (Substation Automation System) more than that no experimental tests or prototypes have been presented. In [9] authors present a prototype based on the current differential principle scheme and IEC61850 implemented in an RTDS (Real Time Digital power system Simulation) and some special 
card for acquisition ,the cost of the presented solution is more expensive comparing to SAS cost which is not economic for the use.

In this paper, we present a novel concept of a smart local backup protection SLBP (Smart Local Backup Protection) used to support and backup of the substation's relays, The SLBP is based on simple logical protective schemes used to protect specific zones of the substation, those logics schemes can be implemented easily in the SAS. The SLBP has been developed using an open access library of protocol IEC61850 and it has been implemented on an industrial computer us ing the free operating system Linux. Moreover, a low cost prototype has been presented for testing the proposed SLBP.

The paper is structured as follow the first section presents the concept of SLBP in a smart substation, the second section describes several logical protection schemes integrated in the SLBP .Results of simulation with the proposed platform are presented in the last section followed by a conclusion.

\section{THE SMART LOCAL BACKUP PROTECTION CONCEPT}

A smart substation is based on advanced new technologies of communication, NCIT (nonconventional instrument transformers) and intelligent primary equipment; those technologies are able to fulfil requirements of reliability, efficiency flexibility, and environmentally friendly operation.

The SAS of smart substation is based on IEC61850 communication standards, the architecture of the SAS called "three-layer-two-network" consists of three levels : the process level, bay level and station level. The process level contains typically intelligent circuit breakers, intelligent sensors, MU (Merging Unit) and NCIT . All those devices are connected by a communication network called the Process Bus. The bay level devices are used for control, monitoring and protection while the station level consists of HMI (Human Machine Interface) and gateways for remote communication with SCADA and other substations.

The process bus is based on both the transmission of SVs (Sampled Values) of current and voltage according to IEC61850-9-2 and the transmission of trips control by the high speed communication of the GOOSE (Generic Object Oriented Substation Event) according to IEC61850-8-1 standards.

The most efficient SAS architecture proposed for smart substations is presented in Figure 1, which consist of a redundant zero switchover network using the PRP (Parallel Redundancy Protocol) protocol in the bay bus [10], [11] and HSR (High-availability Seamless Redundancy) in the process bus.

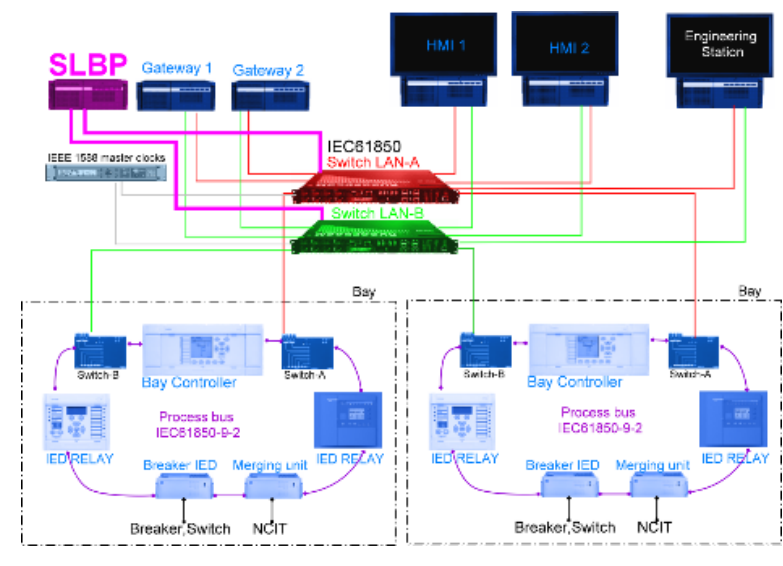

Figure 1. Smart Substation SAS

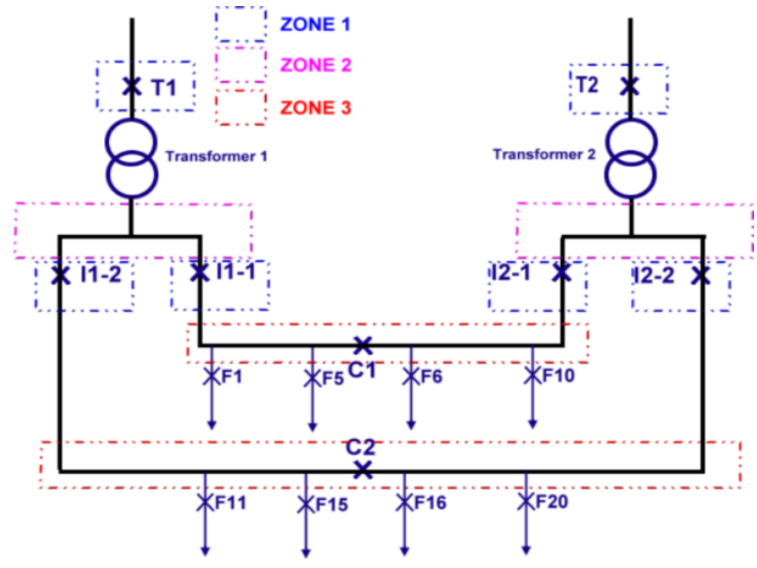

Figure 2. Substation one line diagram and protective zones

The SLBP is a DAN (double Attached Node) devices connected to the bay level. The SLBP uses SVs available from different MU in the process bus and uses data of organ's position coming from IEDs (Intelligent equipment) devices in GOOSEs. The SVs and GOOSEs are used in logical schemes whose outputs are send as GOOSE to IEDs for tripping or interlocking. The advantage of this architecture is that the SLBP will benefit from the redundant network, so any loss of data in one network can be backed up in the other one, which will offers a secure and reliable protection to the substation. The SLBP offers the possibility to implement different protection schemes. In this paper, we propose some simple schemes characterized by efficiency and rapidity that can be implemented in the most of substations. For the sake of a better demonstration of the concept we took the example of substation presented in Figure 2. This substation 
contains 2 transformers (T1, T2), and two switchgears consisting of 4 incomers (I1-2, I1-1, I2-1, I2-2), 2 bus couplers $(\mathrm{C} 1, \mathrm{C} 2)$ and 20 feeders (F1-F20). The Substation can be divided into 4 zones as follow:

- Zone 1: protection of the Circuit breaker.

- Zone 2: Protection of the MV cable between transformer and incomer.

- Zone 3: Protection of the switchgear busbar

- Zone 4 : Protection of primary equipments

The proposed protective relay Schemes suggested for each zone are discussed next.

\section{THE PROTECTIVE RELAY SCHEMES}

\section{a. Cable ground-fault protection}

In order to protect the MV (Medium Voltage) cable linking the transformer and the incomer the following protection scheme is used. If the transformer's relay detects a fault and the incomer's relay didn't detect fault, this means that the fault occurred in the MV cable, thus; the main transformer relay should trip both the circuit breaker of transformer and the incomer in order to isolate the cable. In traditional substations this protective logic is done by wiring the pickup overcurrent protection outputs from the incomer relay to the transformer main relay. If this wire is cut or isn't well connected in the right input of the transformer's relay this can lead to a dysfunction of the tripping logic which can cause severe damage to the MV cable and the circuit breakers. This protective scheme was implemented in SLBP using GOOSE exchange between incomer's relay and transformer's relays as shown in Figure 3. Both transformer's relay and incomer's relay publish a GOOSE after detecting a fault, if the SLBP receives a GOOSE from the transformer's relay and didn't receive a GOOSE from the Incomer's relay then the SLBP send an instantaneous trip GOOSE to transformer and the incomer's relays to isolate the fault.

\section{b. Switchgear busbar protection}

In order to protect the switchgear busbar against internal fault the following protection scheme is used. The operation of this scheme depends on the busbar coupler $\mathrm{CB}$ as follow:

- If the bus coupler circuit breaker is open then:

If the incomer's relay detects a fault and no one of feeders connected to the half busbar of this incomer detect a fault, the fault is considered as an internal fault in the switchge ar busbar so in this case the SLBP must react and send trip orders to open the CB of the incomer and isolate the half busbar.

- If the bus coupler circuit breaker is close:

If the incomer's relay detects a fault and no one of the feeders relays connected to the busbar detect a fault, then the fault is considered in the switchgear busbar, so in this case the SLBP must react and send trip orders to open both circuit breakers of the incomer and bus coupler after 200ms the SLBP recheck if the fault is still present then sends trip order to the circuit breaker of last incomer so that the busbar can be totally isolated. This logic was implemented in the SLBP by using GOOSEs published from the feeders' relay and the incomer's relays, in the case of detecting a fault, which they will be used by the SLBP according to the described logic.

\section{c. Circuit breaker failure protection}

This protection is used to protect a circuit breaker that fails to trip after an order due to a problem in the wire trip order or in the circuit breaker itself. In this case, the circuit breaker must be isolated.

The following logic was implemented in the SLBP in order to protect a CB failure: If a tripping order is sent to a circuit breaker the same order is sent as GOOSE to the SLBP which triggered a counter, then after a defined time, example 300ms, if the SLBP didn't receive a GOOSE indicating the open position of the $\mathrm{CB}$, as a result the SLBP send trip orders to other sources that can feed the fault in order to isolate the failed $\mathrm{CB}$ and clear the fault. Figure 4 presents the logic of a $\mathrm{CB}$ failure.

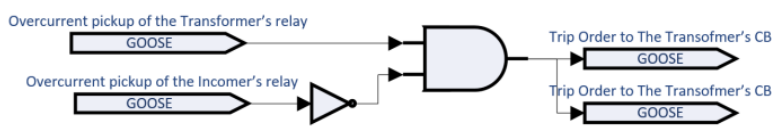

Figure 3. Cable ground-fault protection logic

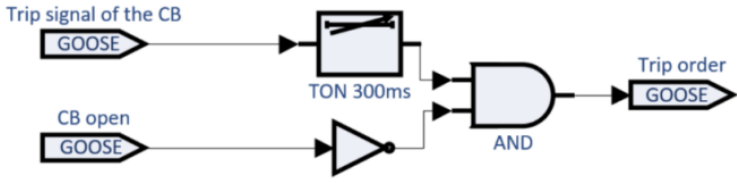

Figure 4. Circuit breaker failure protection logic 


\section{d. Backup function protection}

Using the Sampled Value measurements in process bus, the SLBP can play the role of a Backup function protection of all protective relays existed in the substation, by using the same principle they use. As a demonstration example we use the overcurrent function protection.

The sampled value of current given from MU are used by SLBP and in the same time the SLBP checks the GOOSE of the overcurrent pickup of the protective relay. If the value of the GOOSE is "true" this mains that the protective relay detects the fault and is going to trip after the setting time, in this case the SLBP don't act. If the value of the overcurrent pickup GOOSE is "false" and the SV of the current is over the threshold setting, this mains that the protective relay didn't detect the fault, in this case the SLBP starts counting to $2 \mathrm{Ts} / 3$ and it checks again the value of GOOSE if it sill "false" then the SLBP sends a trip order after counting Ts/3. The total time of tripping is given in (1).

$$
T_{s}=\frac{2 * T_{s}}{3}+\frac{T_{s}}{3}
$$

Where:

Ts: Setting time of tripping overcurrent function

Figure 5 describes the logic of overcurrent backup function protection.

The same Principe can be applied to other protection functions such as overvoltage, under voltage, over frequency and under frequency and more application in [12].

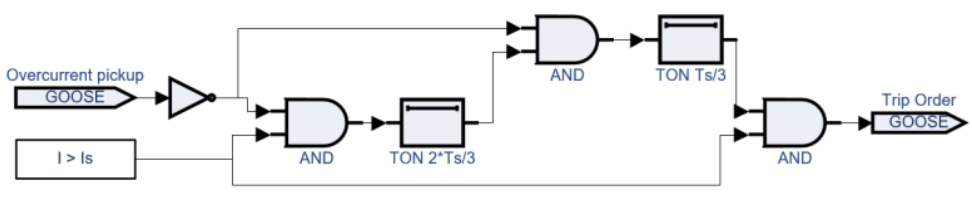

Figure 5. Backup function protection logic

\section{SIMULATION \& RESULTS}

In order to validate the efficiency of the proposed solution, the SLBP was built using an open source library dedicated to the IEC61850 protocol and offers the possibility to publish and subscribe GOOSEs and SVs. Then, the SLBP was deployed on an industrial computer containing the free operating system Linux Ubuntu .For the sake to avoid the packet storm that can happened in the network, the SLBP was programmed to take in consideration only Ethernet packets of GOOSE or SV by filtering the packet types where the GOOSE type is 0x88b8 and the SV type is 0x88ba.

Figure 6 presents the low cost platform used for tests. The platform consists of two numerical protective relays VAMP, a laptop and a switch. The laptop is used for the analysis and which contains the software IEDScout from Omicron this tool allows the analysis of IEC61850 networks and especially to monitor the exchange of GOOSEs.

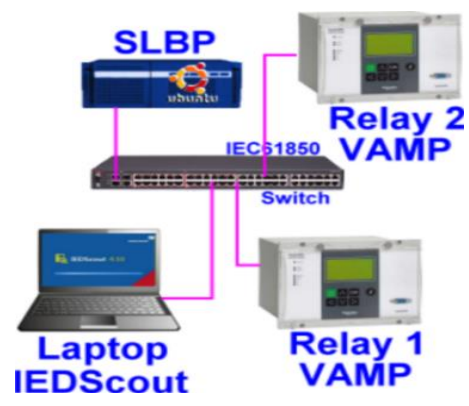

Figure 6. Architecture of the test platform

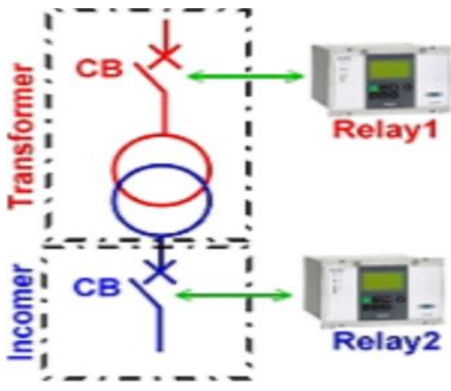

Figure 7. The simulation example of tranformer and incomer 
Using the described platform the three following protection schemes were applied on the example presented in Figure 7:

- Breaker failure protection

- Cable ground-fault protection

- Backup function protection

\section{a. Breaker failure protection}

The fail trip of the incomer's circuit breaker was tested following two scenarios. Scenario 1: The incomer's relay sent an order of trip and the circuit breaker tripped successfully. Scenario 2: The incomer's relay sent an order of trip but the circuit breaker fails to trip and remained close. The captures from IEDScout for the two scenarios are presented respectively in Figure 8 and Figure 9.

\begin{tabular}{|c|c|c|}
\hline a & $20: 38: 11.698338$ & VAMPRELAYZU $\perp$ NOSGOSgCbGOOSE1 \\
\hline & $20: 36: 11.948456$ & VAMPRELAVZLLNDSGOSgcbGOOSE? \\
\hline
\end{tabular}

Figure 8. Scenario 1 IEDScout capture of Breaker failure

\begin{tabular}{c|c|l}
\hline G & $20.38: 15.248563$ & VAMPRELAY2LLLNDSGOSgcbGOOSE1 \\
\hline G & $20.39: 15.553 d 15$ & ELPPIEDLLNDSGO\$gcbGOOSE1
\end{tabular}

Figure 9. Scenario 2 IEDScout capture of Breaker failure

The first capture shows that relay 2 sent a trip order to the incomer's CB and in the same time published a GOOSE GOOSE1 (VAMPRELAY2/LLNO\$GO\$gcbGOOSE1), after the SLBP has received the GOOSE1 by the SLBP it started waiting to receive the change status of the CB, after 250,120ms the CB opened and relay 2 published a second GOOSE indicating the status open of the CB (VAMPRELAY2/LLN0\$GO\$gcbGOOSE2).Finally the SLBP received the GOOSE and as result it didn't act. In the second Scenario the SLBP received GOOSE trip from relay 2 (VAMPRELAY2/LLNO\$GO\$gcbGOOSE1) but after 300ms the SLBP did not receive GOOSE of CB open position, so the SLBP sent GOOSE (SLBPIED/LLNO\$GO\$gcbGOOSE1) tip to relay 1 after $4.852 \mathrm{~ms}$ in order to is olate the failed CB. In the two scenarios the SLBP acted as was described in the CB failure section.

\section{b. Cable ground-fault protection}

In this test the ground-fault protection between the transformer and the incomer was simulated. The capture from IEDScout for the two cases are presented respectively in Figure 10 and Figure 11:

\begin{tabular}{|c|c|c|}
\hline G & 20.59 .58 .516429 & VAMPRELAY1/ШNOSGOSgCbGOOSE1 \\
\hline 6 & 20.59.5A.522166 & 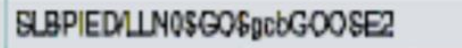 \\
\hline
\end{tabular}

Figure 10. Case 1 IEDScout capture

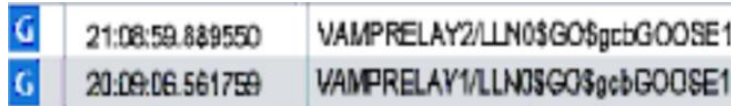

Figure 11.Case 2 IEDScout capture

In the first case, relay 1 detected a fault and sent. GOOSE(VAMPRELAY1/LLN0\$GO\$gcbGOOSE1) to SLBP, but relay 2 didn't detect the fault, so the SLBP sent instantaneous trip GOOSE (SLBPIED/LLNO\$GO\$gcbGOOSE2) to relay 1 and relay 2. In the second case the relay 2 detected a fault and published a GOOSE (VAMPRELAY2/LLNO\$GO\$gcbGOOSE1) after that relay 1 detected fault and published a GOOSE (VAMPRELAY1/LLN0\$GO\$gcbGOOSE), this means that the fault has been detected first by incomers then by the transformer which correspond the normal 
behavior of a fault that occurs in the downstream of the incomers so in this case the SLBP didn't act and wait the normal trip of other relays. In the two cases the SLBP act as it was described in the cable ground-fault protection section.

\section{c. Backup function protection}

In this test other tools were used to publish and visualize sampled value, the software Volcano allows to publish sampled value of current and voltage according to IEC61850-9-2, and the software 61850 Eagle visualize the waveform and the magnitude of the sampled values. Figure 12 shows the waveform of a SV and Figure 13 the Ethernet packet of a SV captured with WireShark software.

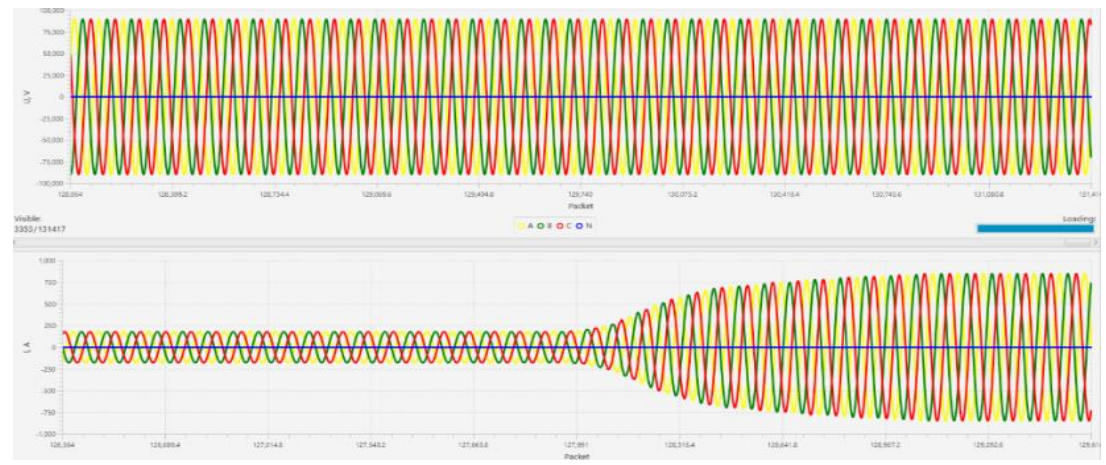

Figure 12.Capture of SV from 61850 Eagle

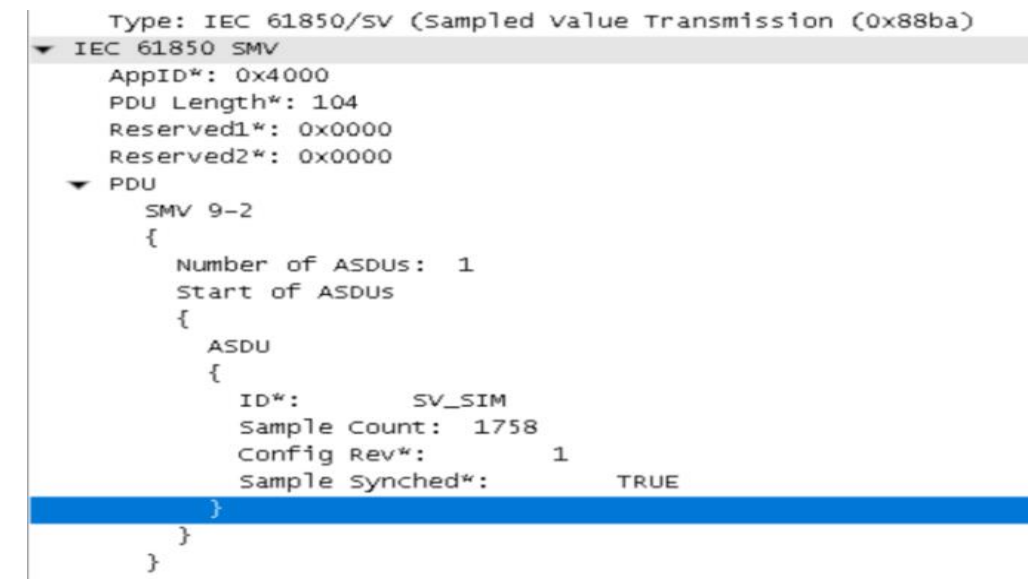

Figure 13.WireShark capture of SV packet

Effectively the type of the Ethernet packet effectively to the SV (0x88ba) and the ID of the published SV is "SV_SIM" which was conFigureured to be subscribed by the SLBP. In this test the backup of an overcurrent function was simulated using the setting given in Table 1.

Table 1. OVERCURRENT SITTING

\begin{tabular}{ll}
\hline Current threshold (A) & Time (s) \\
\hline 500 & 1.5 \\
\hline
\end{tabular}

In order to debug and monitor the operation of the SLBP, we con Figureure 4 GOOSES as follow: GOOSE1: (SLBPIED/LLNO\$GO\$gcbIED1F511G1) this GOOSE is sent when the SLBP detected that the current exceeded 500A. GOOSE2: (SLBPIED/LLN0\$GO\$gcbIED1F511G2) this GOOSE is sent to inform 
that 2Ts/3 is elapsed and trip is in Ts/3. GOOSE3: (SLBPIED/LLNO\$GO\$gcbIED1F511G3) this GOOSE is sent after Ts is elapsed to trip the CB. GOOSE3: (SLBPIED/LLN0\$GO\$gcbIED1F511G4) this GOOSE is sent if the VAMP relay detectzs also the fault and sent it's pickup GOOSE to the SLBP.

The test was done following the two scenarios: Scenario 1: the SV of current over 500A and the Vamp relay didn't detect the fault. Scenario 2: the SV of current over 500A and the vamp relay detected the fault after 500ms. The capture from IEDScout of the two scenarios is given in Figure 14 and Figure 15.

\begin{tabular}{|c|c|}
\hline $21: 14: 46.515281$ & 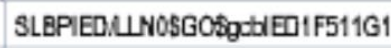 \\
\hline $21: 14: 47.571390$ & 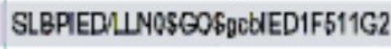 \\
\hline 21:14:48:024687 & SLBPIED:UNOSGO\$gCDIED 1 F511GS \\
\hline
\end{tabular}

Figure 14.Overcurrent function IEDScout capture scenario1

\begin{tabular}{|c|c|c|}
\hline & 21:33:12:652134 & 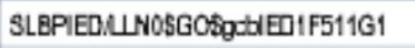 \\
\hline & 21.39:11.721239 & VAMPRELAY1/LLNDSGO\$ \\
\hline & 21:33:11.T\$E223 & SLBPIED,UNOSGO\$9CDEDIF511G4 \\
\hline
\end{tabular}

Figure 15.Overcurrent function IEDScout capture scenario2

In the first case when the current was over 500A the SLBP sent GOOSE 1 and after $\mathrm{t} \_1=\left(2 * \mathrm{~T} \_\mathrm{s}\right) / 2=1.002511 \mathrm{~s}$ the SLBP didn't receive any pickup overcurrent GOOSE from the transformer's relay, so it sent GOOSE 2 to inform that a trip will occurred in Ts/3. After 500ms, the SLBP sends GOOSE 3 to trip the CB. The total time between detecting I>500A and sending tripping GOOSE3 is equal to 1,505806s which corresponds to the setting of overcurrent relay. The SLBP has successfully executed its function.

In the second case was over 500A the SLBP sent GOOSE 1 but after 1,069099s it received a pickup overcurrent GOOSE from VAMP relay the SLBP stop the program and send GOOSE4, also in this case the SLBP has successfully executed its function. The test results show that the proposed protection schemes are able to effectively improve the security and reliability of protection as well as reduce risk of equipment's failure and protection's mal operations.

\section{d. Advantages of SLBP over other solutions}

The proposed architecture, protection schemes and testing results prove that the SLBP has the following advantages:

- The SLBP employs simple logic schemes which guarantee the good operation better than complex algorithm such as current differential that requires high speed acquisition and time synchronization precision.

- Comparing with Wide Area backup protection WABP, the amount of data required by the SLBP is less important. Moreover, in the case of the SLBP all data are present in the same location and in a redundant network which make data more controllable, which is not the case in WABP that requires data from different substations and that make the probability to lose data greater.

- In the SLBP the proposed protections schemes can be applied in most of substations as they protect the hall substation and can be divided into simple specific zones, which is more difficult and requires a good knowledge of the electrical grid in the case of WABP.

- The price of the SLBP is cheaper comparing with other solutions as the SLBP is based on free development library and free operating system.

- The SLBP can be tested independently from the substation, as we did in our tests, because GOOSEs and SVs can be simulated using free tools in order to verify the good operation of SLBP and after validation it can be integrated in the substation which will reduce the cost of commissioning and also reduce the risk of mal operation

\section{CONCLUSION}

The proposed smart local backup protection presents more security to the substation, by preventing catastrophic damage caused by communication faults or defects of substation automation system equipment's. In this paper, we present how easily the SLBP can be implemented in the SAS using GOOSEs 
and SVs from different IEDs; also, a low cost simulation platform has been presented to be sure of the operation of the protection schemes deployed in the SLBP. We present three logical protection schemes, but many other protection schemes can be implemented using the same principle which will make the object of our future works. Eventually testing result demonstrates that the SLBP can be recognized as an enhancement of the future backup protection in the smart substation.

\section{REFERENCES}

[1] D. Novosel, M. M. Begovic, and V. Madan, "Shedding Light on Blackouts", IEEE power \& energy magazine, Jan. \& Feb. 2004.

[2] Z. Q. Bo et al., "Novel architecture for integrated wide area protection and control," Power Engineering Conference (UPEC), 2015 50th International Universities, Stoke on Trent, 2015, pp. 1-4.

[3] Y. Lv, D. You, K. Wang, L. Wang, Z. Chen and S. Huo, "Study on wide-area backup protection system for the smart grid, "Electric Utility Deregulation and Restructuring and Power Technologies (DRPT), 2011 4th International Conference on, Weihai, Shandong, 2011, pp. 218-224.

[4] L. Chen, K. Zhang, Y. Xia and G. Hu, "Study on the Substation Area Backup Protection in Smart Substation," 2012 Asia-Pacific Power and Energy Engineering Conference, Shanghai, 2012, pp. 1-4.

[5] A Jusoh, T Sutikno, TK Guan, S Mekhilef, "A Review on favourable maximum power point tracking systems in solar energy application," TELKOMNIKA Telecommunication Computing Electronics and Control., vol. 12, no. 1, pp. 6-22, 2014.

[6] Q. Li, Z. Zhou, D. Du, Z. Li, W. Li and X. Wang, "A novel substation area backup protection for smart substation," 2013 IEEE PES Asia-Pacific Power and Energy Engineering Conference (APPEEC), Kowloon, 2013, pp. 1-4.

[7] S.N.Nikolovski et al. "Protection Coordination and Anti Islanding Protection Solution for Biomass Power Plant Connected on Distribution Network". International Journal of Electrical and Computer Engineering., vol 6, no 6, pp. 2526-2537, 2016.

[8] A.N.Sarwade et al. "Reach and Operating Time Correction of Digital Distance Relay". International Journal of Electrical and Computer Engineering. Vol 7, No 1.

[9] Y. Liu; H. Gao; W. Gao; F. Peng, "Development of a Substation-Area Backup Protective Relay for Smart Substation," in IEEE Transactions on Smart Grid, vol.PP, no.99, pp.1-10

[10] H. Kirrmann, K. Weber, O. Kleineberg and H. Weibel, "Seamless and low-cost redundancy for substation automation systems (high availability seamless redundancy, HSR)," 2011 IEEE Power and Energy Society General Meeting, San Diego, CA, 2011, pp. 1-7.

[11] Naiara Moreira, Elías Molina, Jesús Lázaro, Eduardo Jacob, Armando Astarloa, Cyber-security in substation automation systems, Renewable and Sustainable Energy Reviews, Volume 54, February 2016, Pages 1552-1562.

[12] M.N.DAZAHRA et al. "Decentralized control of substations in smart cities". Advances in Ubiquitous Networking 2: Proceedings of the UNet' 16 